\title{
AVALIAÇÃO DA QUALIDADE DA ATENÇÃO À SAÚDE DE ADOLESCENTES NO PRÉ-NATAL E PUERPÉRIO
}

\author{
Evaluation of quality of health care for adolescents in the prenatal and puerperium \\ Evaluación de la calidad de atención a la salud de adolescentes en el prenatal y en el \\ puerperio
}

\section{RESUMO}

Pesquisa avaliativa que objetivou avaliar a qualidade da atenção pré-natal e puerperal a adolescentes com filhos nascidos vivos em instituição pública de saúde de Teresina, Piauí. Foram utilizados dois parâmetros para avaliar o cuidado pré-natal: um índice internacional (Adequacy of Prenatal Care Utilization) e outro nacional, baseado em recomendações do Ministério da Saúde. A qualidade da atenção foi categorizada em adequada superior, adequada, intermediária e inadequada. Identificou-se que mais da metade das mulheres iniciou o pré-natal precocemente e o número de consultas de pré-natal foi inadequada. A maioria realizou exames de pré-natal de rotina, $75 \%$ tiveram as mamas examinadas e $88,6 \%$ foram orientadas sobre aleitamento materno. A atenção puerperal foi intermediária para 38,6\% das mulheres, 52,3\% não retornaram à unidade de saúde e tampouco receberam visita domiciliar, $70,5 \%$ foram orientadas sobre os métodos contraceptivos e 93,2\%, sobre aleitamento materno. Esforços devem ser empreendidos para garantir a qualidade da atenção no ciclo gravídico-puerperal.

Palavras-chave: Avaliação em saúde. Gravidez na adolescência. Enfermagem. Cuidado pré-natal. Puerpério

\begin{abstract}
It is an evaluative study aimed to assess the quality of prenatal and postpartum for adolescents with children born alive in a public health institution in Teresina, Piauí. Two parameters were used to evaluate the prenatal care: an international index (Adequacy of Prenatal Care Utilization) and a national one, based on recommendations from the Ministry of Health. The quality of care was categorized as more appropriate, adequate, intermediate and inadequate. It was noticed that more than half of the women began the prenatal care at an early stage and the number of visits for prenatal care was inadequate. Most of them performed the ordinary prenatal exams, $75 \%$ had their breasts examined and $88.6 \%$ were advised about breastfeeding. The postpartum care was intermediate to $38.6 \%$ of women, $52.3 \%$ did not return to the health unit, nor received home visit, $70.5 \%$ were advised about contraceptive methods and $93.2 \%$ on breastfeeding. Efforts should be undertaken to ensure the quality of care in pregnancy up to the puerperium.
\end{abstract}

Keywords: Health Evaluation. Teenage Pregnancy. Nursing. Prenatal Care. Childcare.
Resumen

Investigación evaluativa que tuvo como objetivo evaluar la calidad de la atención prenatal y cuidado de los hijos de padres adolescentes nacidos vivos en institución de salud pública en Teresina, Piauí. Se han utilizado dos parámetros para evaluar la atención prenatal: un índice internacional (Adequacy of Prenatal Care Utilization) y otro nacional, con base en las recomendaciones del Ministerio de la Salud. La calidad de la atención fue categorizada como más apropiada, adecuada, intermedia e inadecuada. Se identificó que más de la mitad de las mujeres comenzaron a recibir atención prenatal antes del tiempo y el número de visitas de atención prenatal era inadecuado. La mayoría realizó examen prenatal de rutina, el $75 \%$ tuvieron sus pechos examinados y $88,6 \%$ se les aconsejó sobre la lactancia materna. La atención de posparto fue intermediaria al 38,6\% de las mujeres, $52,3 \%$ no regresaron a la clínica o bien recibieron las visitas domiciliarias, el $70,5 \%$ se les aconsejó sobre los métodos anticonceptivos y el 93,2\% sobre la lactancia materna. Se deben hacer esfuerzos para garantizar la calidad de la atención durante el embarazo y el parto.

Palabras-clave: Evaluación en Salud. Embarazo en Adolescencia. Enfermería. Atención Prenatal. Puerperio.

\footnotetext{
${ }^{1}$ Mestre em Ciências e Saúde. Universidade Federal do Piauí - UFPI. Professora Assistente I da Associação de Ensino Superior do Piauí - AESPI. Teresina-PI. Brasil. E-mail: lilianvilarinho@hotmail.com; ${ }^{2}$ Doutora em Enfermagem. Professora Associada da UFPI. Departamento de Enfermagem. Programa de Pós-Graduação em Enfermagem e em Ciências e Saúde da Universidade Federal do Piaú́-UFPI. Teresina - PI. Brasil. E-mail: lidyatn@gmail.com;' Doutora em Saúde Coletiva. Universidade Estadual de Campinas - UNICAMP. Enfermeira do Hospital Universitário Regional de Maringá. Universidade Estadual de Maringá.Maringá-PR. Brasil. E-mail: eeinagahama@uem.br
} 


\section{INTRODUÇÃO}

Cerca de 14 milhões de adolescentes tornam-se mães a cada ano, o que equivale a mais de $10 \%$ dos nascimentos no mundo. Nos países em desenvolvimento, este percentual representa $90 \%$ dos nascimentos, sendo os países da África os que exibem as taxas mais altas, seguidos da Índia, países da América-Latina e Caribe. ${ }^{1}$

No Brasil, sete em cada cem adolescentes entre $15 \mathrm{e}$ 19 anos tornaram-se mães em 2004². Dados recentes do Ministério da Saúde ${ }^{3}$ apontaram que, apesar da redução de $30 \%$ dos partos em adolescentes nos últimos dez anos, em 2009 foram realizados mais de quatrocentos mil partos na rede pública de saúde em todo o território nacional. No Piauí, a redução de partos em adolescentes foi ainda mais significativa $(43,51 \%)$ embora, em 2009, mais de nove mil adolescentes tenham se tornado mães.

A crescente vulnerabilidade desse grupo tem gerado a preocupação crescente de diversos setores da sociedade com o fenômeno da gravidez na adolescência e a implementação de políticas e programas voltados para esta faixa etária.

Apesar da melhoria ao acesso às políticas de prevenção e orientação sobre saúde sexual e reprodutiva, disponibilização gratuita de métodos contraceptivos e aumento da cobertura da Estratégia Saúde da Família (ESF), a qualidade da atenção no ciclo gravídico puerperal necessita ser aprimorada, sendo objeto de estudo em diversas regiões do país. ${ }^{4-5}$

Alguns estudos destacam a importância da atenção pré-natal de qualidade para a redução da morbimortalidade materna e perinatal, especialmente o componente neonatal, visto que este reflete as condições da gravidez, do parto e da assistência perinatal. ${ }^{4-5}$

A avaliação é uma estratégia que contribui para direcionar ou redirecionar a execução de ações e atividades, colaborando para a tomada de novas decisões e melhoria da qualidade do processo assistencial. ${ }^{6}$

Quando se aborda a avaliação da qualidade no setor saúde, é importante destacar os estudos de Avedis Donabedian, o primeiro a propor um referencial teórico sistêmico - a tríade estrutura, processo e resultado, para analisar os serviços de saúde e as práticas assistenciais, sendo a avaliação do processo o reflexo da qualidade da atenção à saúde. ${ }^{7}$ Neste contexto, o presente estudo objetiva avaliar a qualidade da atenção pré-natal e puerperal a adolescentes com filhos nascidos vivos em instituição pública de saúde de Teresina, Piauí.

\section{METODOLOGIA}

Trata-se de uma pesquisa avaliativa, com delineamento transversal, com enfoque na avaliação de processo e da qualidade da atenção pré-natal e puerperal.
Foi conduzida mediante pesquisa em prontuários e entrevistas com adolescentes que deram a luz em uma unidade integrada de saúde pertencente à rede pública municipal de Teresina-PI, no período de 01 de janeiro a 31 de dezembro de 2008.

Teresina tem uma área territorial de $1.176 \mathrm{~km}^{2}$, situa-se na região centro-nor te do estado e meio-norte do nordeste brasileiro, com população residente de 814.439 habitantes, sendo o município mais populoso do Piauí, com a maior parte da infra-estrutura administrativa do Estado. ${ }^{8}$ A capital é considerada polo de saúde, pela quantidade de serviços que oferece tanto na rede pública como na rede privada, e por receber grandes demandas do interior do estado. Abrange três Regionais de Saúde: Centro-Norte, Sul e Leste-Sudeste, com aproximadamente 223 equipes da ESF, 69 Centros de Saúde, uma maternidade estadual de referência, quatro hospitais-maternidades e duas Unidades Mistas de Saúde no setor público municipal que oferecem assistência pré-natal vinculadas à ESF e que atendem pelo SUS. ${ }^{9}$

Para o cálculo do tamanho amostral, considerou-se o número de partos SUS ocorridos na referida instituição no ano de 2008, de acordo com o Sistema Nacional de Nascidos Vivos (SINASC). Obteve-se o total de 1.047 casos, o que correspondeu a 7,5\% dos nascimentos do município de Teresina em 2008. A consulta ao sistema de informação da unidade de estudo permitiu o levantamento de 239 nascidos vivos de adolescentes.

Foram incluídas no estudo as gestantes adolescentes, residentes em Teresina, que realizaram pré-natal nas unidades de saúde deste município e cujo parto foi realizado na maternidade da instituição de saúde em estudo no ano de 2008. Foram excluídas aquelas procedentes de outros municípios (26) e cujo prontuário não tinha registro de realização do pré-natal ou não haviam realizado pré-natal (38).

Os dados foram obtidos por meio da análise de 174 prontuários e entrevista com 44 adolescentes (30\% desses prontuários), durante visita domiciliar. Utilizou-se a fórmula para o cálculo amostral de populações finitas ( $n$ e" N. Za $a^{2}$.p.q/ $\left.(\mathrm{N}-1) \mathrm{d}^{2}+\mathrm{Za}^{2} \cdot \mathrm{p} . \mathrm{q}\right)$, no qual o grau de confiabilidade adotado foi $95 \%$ e o erro amostral foi de $5 \%$. Vale destacar que as entrevistas foram realizadas apenas com o intuito de complementar os dados não registrados nos prontuários, e com as adolescentes localizadas no período da coleta de dados.

Dois instrumentos com perguntas fechadas foram aplicadas entre os meses de março a julho de 2010.0 primeiro foi utilizado para a coleta de dados referente à atenção prénatal em prontuário, e o segundo, utilizado na entrevista, englobava informações complementares sobre a atenção prénatal e puerperal. A elaboração destes instrumentos foi baseada nas recomendações do Ministério da Saúde para atenção prénatal e puerperal qualificada e humanizada nos serviços de saúde. ${ }^{10}$ 


\section{Avaliação do processo do cuidado pré-natal}

As variáveis de estudo foram: número de consultas de pré-natal, idade gestacional do início do pré-natal em semanas, número de exames laboratoriais de rotina realizados, procedimentos clínicos obstétricos em todas as consultas de pré-natal, número de exames de ultrassonografias realizadas e orientação sobre aleitamento materno.

Os parâmetros utilizados para avaliar o pré-natal foram o índice Adequacy of Prenatal Care Utilization (APNCU) ${ }^{11}$ e índice baseado nas recomendações do Ministério da Saúde (índice MS). ${ }^{12}$

0 índice APNCU caracteriza a adequação da utilização do cuidado pré-natal em duas dimensões independentes e distintas:

a) Adequação do início do cuidado pré-natal ou mês em que o cuidado pré-natal foi iniciado: considera que quanto mais precoce, mais adequado o cuidado pré-natal. Estabelece a distribuição da gestação em quatro grupos: meses 1 e 2; 3 e 4; 5 e 6; e 7 a 9 . A adequação do início do cuidado pré-natal corresponde a: ótimo: $1^{\circ}$ ou $2^{\circ}$ mês; adequado: $3^{\circ}$ ou $4^{\circ}$ mês; intermediário: $5^{\circ}$ ou $6^{\circ}$ mês; inadequado: $7^{\circ}$ mês ou mais tarde, ou sem assistência prénatal.

b) Adequação dos cuidados recebidos ou porcentagem de visitas recebidas ajustadas para o mês de início da assistência pré-natal e para a idade gestacional da ocorrência do parto. A medida da adequação dos cuidados recebidos corresponde à razão entre o número de visitas recebidas do número esperado de visitas. 0 número esperado de visitas é baseado no padrão de visitas pré-natais para gestações não complicadas recomendado pelo American College of Obstetricians and Gynecologistis (ACOG): uma visita por mês até 28 semanas, duas visitas por mês até 36 semanas e uma visita por semana até o nascimento. 0 cuidado recebido foi classificado de acordo com sua adequação (proporção do número de visitas recomendado pelo ACOG recebidas desde o início do cuidado pré-natal até o término da assistência prénatal ou nascimento), como se segue: ótimo, maior ou igual 110\%; adequado, 80-109\%; intermediário, 50$79 \%$; inadequado, menos que $50 \%$.

Dessa forma, o APNCU combina a adequação do início da assistência pré-natal (mês de início da assistência) e a adequação da utilização do cuidado pré-natal (número de consultas realizadas) como: ótimo, cuidado pré-natal iniciado até o $4^{\circ}$ mês e realização de $110 \%$ ou mais das visitas recomendadas; adequado, cuidado pré-natal iniciado até $04^{\circ}$ mês e realização de 80-109\% das visitas recomendadas; intermediário, cuidado pré-natal iniciado até o $4^{\circ}$ mês e realização de 50-79\% das visitas recomendadas; inadequado, cuidado pré-natal iniciado após o $4^{\circ}$ mês ou realização de menos que $50 \%$ das visitas recomendadas.

0 índice $\mathrm{MS}^{12}$ combina: 1. idade gestacional de início do pré-natal; 2. número de consultas de pré-natal; 3. realização de procedimentos clínicos obstétricos. Os autores recomendam seis parâmetros:

1. Início do atendimento pré-natal: antes de 14 semanas de gestação.

2. Número de consultas de pré-natal: realização de seis ou mais consultas para uma gestação de 37 semanas ou mais; cinco consultas para a gestação com 32 a 36 semanas; e quatro consultas para gestação com 22 a 32 semanas.

3. Realização de todos os exames de rotina propostos (ABO-Rh, Hb/Ht, Glicemia de Jejum, Urina, VDRL, Anti-HIV e HBsAg).

4. Realização de pelo menos cinco procedimentos clínico-obstétricos em todas as consultas (verificação de peso, PA, BCF, AU e edema).

5. Orientação sobre amamentação.

6. Realização de pelo menos um exame de ultrassonografia.

0 índice $\mathrm{MS}^{12}$ combina a adequação do início da assistência pré-natal, adequação do número de consultas realizadas e realização dos procedimentos clínicoobstétricos, como: adequada superior, quando todas as seis recomendações forem satisfeitas; adequada, com o cumprimento de cinco recomendações; intermediária, na presença de quatro; e inadequada, quando três ou menos recomendações forem satisfeitas.

Cabe ressaltar que a checagem dos procedimentos clínico-obstétricos foi obtida conforme a possibilidade técnica de sua realização, que depende da idade gestacional na época da consulta. Considerou-se que a ausculta dos batimentos cárdio-fetais, mensuração da altura uterina e avaliação da apresentação fetal foram passíveis de realização a partir da segunda metade da gravidez. Por outro lado, cálculo da idade gestacional, aferição da pressão arterial, peso e edema maternos constituíram procedimentos que deveriam ser realizados em qualquer período gestacional e, portanto, em todas as consultas de pré-natal.

\section{Avaliação de processo no puerpério}

As variáveis de estudo foram: realização de consulta de revisão puerperal, visita domiciliar na primeira semana após o parto, avaliação clínico-ginecológica, orientação sobre planejamento familiar e orientação sobre aleitamento materno.

Para estabelecer os critérios de qualidade da atenção puerperal utilizou-se escala de escores que variaram de $0 \mathrm{a}$ 3 pontos, segundo a adequação na realização de cada indicador (Quadro 1). 
Quadro 1 - Indicadores de processo da qualidade na atenção puerperal e respectivos escores.

\begin{tabular}{|c|c|c|c|}
\hline \multicolumn{2}{|r|}{ RDICADOR } & \multicolumn{2}{|c|}{ ETCRE } \\
\hline 1. & Retomo a UBS no pós-parto au visita domiciliar na primeira semana após o parto & Simt 3 & Năo:0 \\
\hline 2. & Avalią̃a clínico-ginecológica na consulta puerperal & Simt 2 & Na:0 \\
\hline 3. & Orientar ôes sobre aletamento materno & Simt 2 & Na:0:0 \\
\hline 4. & Orientações sobre planģamento familiar & Simt 1 & Na:0:0 \\
\hline
\end{tabular}

Com a finalidade de analisar a qualidade da atenção ao puerpério foram considerados todos os escores e calculado 0 somatório obtido individualmente em cada item, que variou de um escore mínimo de zero até escore máximo de oito. Desta forma, determinaram-se quatro níveis de qualidade conforme as escalas somadas de classificação: inadequada: de 0 a 2 pontos; intermediária: de 3 a 4 pontos; adequada: 5 a 6 pontos; adequada superior. 7 a 8 pontos.

Os dadosforam digitadoseanalisadosno programaSPSSversão 18.0. Foram calculadas as distribuições de frequência das variáveis relacionadasaopré-natal,epuerpério. 0 projeto de pesquisa foi aprovado pelo Comitê de Ética em Pesquisa (CEP) da Universidade Federal do Piauí (UFPI), CAAE nº 0228.0.045.000-09.

\section{RESULTADOS}

Quanto à idade gestacional de início da assistência, em relação ao critério APNCU, a assistência foi considerada adequada superior para 18,2\% das gestantes; adequada para 59,1\%; intermediária para 20,4\% e inadequada para 2,3\% das mulheres. Em comparação com o índice MS ${ }^{12}, 63,6 \%$ das gestantes tiveram classificação adequada e 36,4\%, inadequada (Tabela 1).

Considerando-se onúmero de consultas de pré-natal, observouseque, de acordo como critério APNCU, ocuidado pré-natalfoiintermediánio para $52,3 \%$, inadequado para $43,2 \%$ e adequado para $4,5 \%$. Por outro lado, conforme critérios do índice MS ${ }^{12}$, o cuidado recebido foi classificado como adequado para $75 \%$ das adolescentes e inadequado para $25 \%$. Considerando-se o sumário do índice de adequação (APNCU e MS), 0 cuidado pré-natal foi inadequado para 22,7\% das gestantes (Tabela 1).

Em relação aos exames de rotina, todas as gestantes realizaram tipagem sanguínea (ABO/Rh), glicemia dejejum, uninatipo I, hemoglobina ehematócrito. OexamedeVDRLfoi realizado por $74,7 \%$ das adolescentes e o teste Anti-HIV, por 69,0\% (Tabela 2).

Considerando-se a aplicação das doses da vacina antitetânica, $6,3 \%$ das gestantes receberam apenas uma dose; $19,5 \%$, duas; $35,6 \%$, três; $16,7 \%$ receberam o reforço; e $8,6 \%$ já estavam imunizadas.

Acerca dos demais exames, $68,2 \%$ realizaram a citologia oncótica; 50,0\% fizeram duas ultrassonografias; e 31,8\% conseguiram pelo menos uma. Quanto ao exame das mamas, observou-se que $75,0 \%$ das adolescentes tiveram as mamas examinadas e $88,6 \%$ afirmaram ter recebido orientações sobre aleitamento materno durante as consultas. Entretanto, 81,8\% não receberam informação sobre o tipo de parto durante o pré-natal.

Em relação à realização dos procedimentos clínicos obstétricos nas consultas de pré-natal, verificou-se que $56,8 \%$ foram realizados de três a quatro procedimentos. No tocante ao profissional de saúde que realizou o pré-natal, registrou-se que $61,4 \%$ das adolescentes efetuaram 0 acompanhamento prénatal com o mesmo profissional.

No puerpério, 52,3\% das adolescentes não retornaram à UBS até $042^{\circ}$ dia de pós-parto para consulta de revisão e não receberam visita domiciliar na primeira semana pós-parto. 0 percentual de 70,5\% recebeu orientações sobre os métodos de anticoncepção e 93,2\% receberam orientações, ainda na maternidade, sobre aleitamento materno (Tabela 3). A atenção puerperal foi qualificada como adequada superior para $36,4 \%$ das mulheres, intermediária para $38,6 \%$ das adolescentes, inadequada para 18,2\% eadequada para 6,8\% (Gráfico 1).

Tabela 1. Adequação da atenção pré-natal das adolescentes entrevistadas de acordo com o índice APNCU e índice proposto por Almeida e Barros (2005), Teresina (PI), Brasil, 2008.

\begin{tabular}{|c|c|c|c|c|}
\hline \multirow[t]{2}{*}{ Gitóio de Mai } & \multicolumn{2}{|c|}{ Indice APAn } & \multicolumn{2}{|c|}{ hidice is } \\
\hline & $\mathrm{N}$ & 听 & $n$ & $\sigma_{0}$ \\
\hline \multicolumn{5}{|l|}{ IG no írício do PN } \\
\hline Adequada superior & 08 & 182 & - & - \\
\hline Adequada & 26 & 59,1 & 28 & 63,6 \\
\hline Intermediaria & 09 & 20,4 & - & - \\
\hline Inadequada & 01 & 23 & 16 & 36,4 \\
\hline \multicolumn{5}{|l|}{$N^{\circ}$ de consultios de $P N$} \\
\hline Adequada superior & $\infty$ & Q0 & - & - \\
\hline Adequada & QR & 4,5 & 33 & 75,0 \\
\hline Intermediária & 23 & 523 & - & - \\
\hline Inadequada & 19 & 43,2 & 11 & 25,0 \\
\hline \multicolumn{5}{|l|}{ Adequarāo do PN } \\
\hline Adequada superior & $\infty$ & 00 & 04 & 9,1 \\
\hline Adequada & QR & 4,6 & 17 & 38,6 \\
\hline Intermediária & 32 & 727 & 13 & 20,6 \\
\hline Inadequada & 10 & 227 & 10 & 22,7 \\
\hline Total & 44 & 100 & 44 & 100 \\
\hline
\end{tabular}


Qualidade do pré-natal e puerpério de adolescente

Tabela 2. Distribuição das adolescentes segundo as variáveis de atenção pré-natal, segundo registro nos prontuários amostrados, Teresina (PI), 2008.

\begin{tabular}{|c|c|c|}
\hline Variávés de atenç̄opénatal & H & $\mathbf{q}$ \\
\hline \multicolumn{3}{|l|}{ Hímerode cansuliso* } \\
\hline 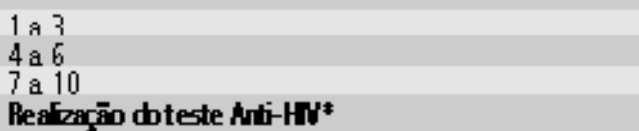 & $\begin{array}{l}73 \\
83 \\
68\end{array}$ & $\begin{array}{l}13.7 \\
47.7 \\
39.1\end{array}$ \\
\hline Sim & $\begin{array}{l}170 \\
25 \\
29\end{array}$ & $\begin{array}{l}69.0 \\
14.3 \\
16.7\end{array}$ \\
\hline 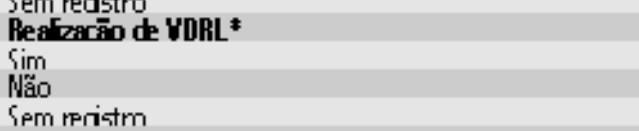 & $\begin{array}{l}130 \\
06 \\
78\end{array}$ & $\begin{array}{r}74.7 \\
3.4 \\
21.8\end{array}$ \\
\hline 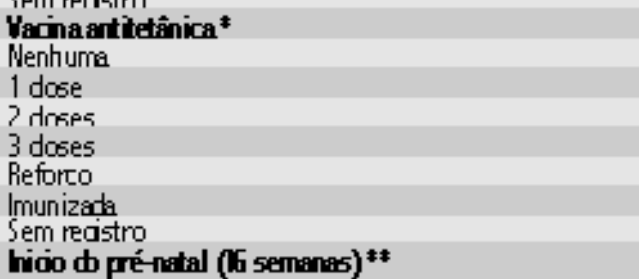 & $\begin{array}{l}03 \\
11 \\
34 \\
62 \\
29 \\
15 \\
20\end{array}$ & $\begin{array}{r}1.7 \\
6.3 \\
19.5 \\
35.6 \\
16.7 \\
8.6 \\
11.5\end{array}$ \\
\hline $\begin{array}{l}\text { 1 a } 8 \\
9 \text { a } 16 \\
17 \text { ou mais } \\
\text { Rea'zaçio de dologgia oncatica }{ }^{* *}\end{array}$ & $\begin{array}{l}06 \\
28 \\
10\end{array}$ & $\begin{array}{l}13.6 \\
63.6 \\
22.7\end{array}$ \\
\hline $\operatorname{Sim}_{\text {Nă }}$ & $\begin{array}{l}30 \\
14\end{array}$ & $\begin{array}{l}68.7 \\
31.8\end{array}$ \\
\hline He ulassonografis (US) $* *$ & & \\
\hline $\begin{array}{l}1 \\
? \\
\text { ou mais }\end{array}$ & $\begin{array}{l}14 \\
72 \\
08\end{array}$ & $\begin{array}{l}31.8 \\
5,1.0 \\
18.2\end{array}$ \\
\hline Exame das mamas** & & \\
\hline 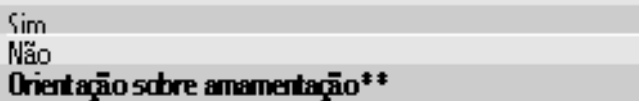 & $\begin{array}{l}37 \\
11\end{array}$ & $\begin{array}{l}75.0 \\
25.0\end{array}$ \\
\hline $\operatorname{sim}_{\text {Nắ }}$ & $\begin{array}{l}39 \\
05\end{array}$ & $\begin{array}{l}88.6 \\
11.4\end{array}$ \\
\hline Oriertap̄oschre tipo de pato noprénatal ** & & \\
\hline $\begin{array}{l}\text { Sim } \\
\text { Năo } \\
\text { Cansulbs con omesmo profission: ** }\end{array}$ & $\begin{array}{l}08 \\
36\end{array}$ & $\begin{array}{l}18.7 \\
81.8\end{array}$ \\
\hline 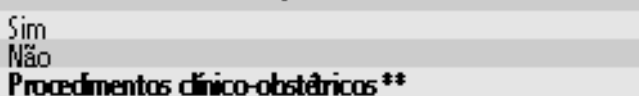 & $\begin{array}{l}27 \\
17\end{array}$ & $\begin{array}{l}61.4 \\
38.6\end{array}$ \\
\hline $\begin{array}{l}\text { 1. } \text { ค } \\
\text { 3a } 4 \\
5\end{array}$ & $\begin{array}{l}03 \\
25 \\
16\end{array}$ & $\begin{array}{r}5.8 \\
56.8 \\
36.3\end{array}$ \\
\hline Exames de rdina realizacts ** & & \\
\hline $\begin{array}{l}1 \text { a } 2 \\
3 \text { a } 4 \\
5 \text { a } 7 \\
\text { Todos }\end{array}$ & $\begin{array}{l}00 \\
05 \\
11 \\
28\end{array}$ & $\begin{array}{r}0.0 \\
11.4 \\
25.0 \\
63.6\end{array}$ \\
\hline
\end{tabular}

Tabela 3. Distribuição de mães adolescentes segundo as variáveis de atenção puerperal, Teresina (PI), Brasil, 2008.

\begin{tabular}{|c|c|c|}
\hline & $\mathbf{H}$ & $y$ \\
\hline \multicolumn{3}{|c|}{ 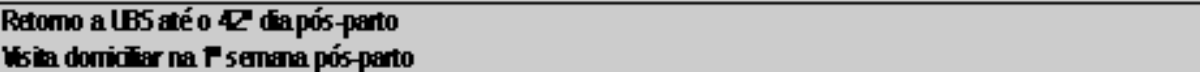 } \\
\hline Sim & 21 & 47,7 \\
\hline Não & 23 & 523 \\
\hline \multicolumn{3}{|c|}{ Oiertaçāo sobre aticoncepp } \\
\hline Sim & 31 & 70,5 \\
\hline Não & 13 & 29,5 \\
\hline \multicolumn{3}{|c|}{ Oientacīo sobre amimentacīo } \\
\hline Sim & 41 & 93,2 \\
\hline Não & 03 & 6,8 \\
\hline Tot:al & 44 & 100,0 \\
\hline
\end{tabular}


Gráfico 1. Distribuição percentual da atenção puerperal de acordo com as escalas somadas de classificação, Teresina (PI), Brasil, 2008

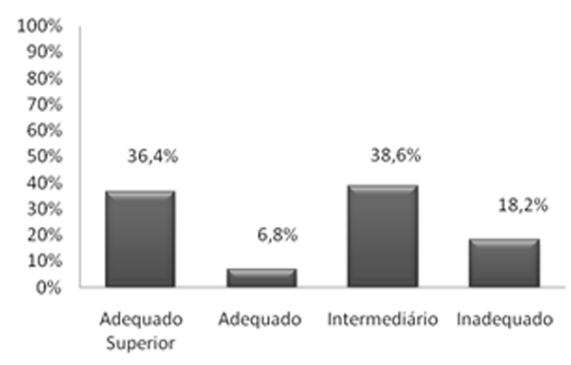

\section{DISCUSSÃO}

Em relação à idade gestacional de início do pré-natal, verificou-se que, de acordo com os dois índices, a atenção prénatal foi considerada adequada para mais da metade das mulheres, ou seja, mais da metade das mulheres iniciou o atendimento pré-natal precocemente. Em relação ao número de consultas, o índice APNCU aponta uma porcentagem pequena de mulheres que obteve assistência adequada em contraste com o obtido pelo índice MS. ${ }^{12}$

Esta divergência na adequação do número de consulta de pré-natal é atribuída ao fato de que, pelo índice APNCU, 0 número esperado de consultas é ajustado para a idade gestacional de início e do término da assistência (momento do parto), o que não acontece ao se utilizar o índice MS, que considera apenas a idade gestacional da ocorrência do parto. ${ }^{12}$

Chama atenção neste estudo a divergência de classificação da adequação da utilização do cuidado pré-natal ao se comparar os dois índices. Este resultado também foi encontrado em estudo realizado em Maringá, no Paraná, que avaliou o processo de atenção pré-natal utilizando o índice APNCU e critérios recomendados pelo PHPN. ${ }^{13}$

Contudo, a aplicação de dois critérios de avaliação da utilização do cuidado torna-se útil, pois permite mensurar se a inadequação na atenção foi atribuída ao número inadequado de consultas ou ao início tardio da assistência. Neste estudo, a inadequação no cuidado foi atribuída ao número insuficiente de consultas de pré-natal e não à IG no início do pré-natal, pois o índice de inadequação desta variável (idade gestacional de início do pré-natal) foi pequeno segundo o APNCU.

A frequência adequada ao pré-natal previne a ocorrência de complicações da gravidez, especialmente na adolescência, o que reafirma a relevância da atenção às adolescentes como questão de saúde pública. A frequência inadequada ao pré-natal entre adolescentes constitui objeto de investigação de outros autores. Um estudo realizado em Montes Claro, Minas Gerais, apontou os motivos relatados pelas gestantes para o número insuficiente de consultas: medo de procedimentos obstétricos, vergonha dos pais, abordagens sobre práticas sexuais.

É importante ressaltar que o índice MS ${ }^{12}$, além da IG no início do pré-natal e número de consultas, considera outros critérios de qualidade baseado em recomendação do MS para estabelecer a adequação do pré-natal. Sendo assim, não foi possivel estabelecer comparação entre os dois índices para avaliar o sumário de adequação do pré-natal.

Uma parcela das adolescentes iniciou o pré-natal tardiamente. Estes resultados também foram encontrados em outros estudos que abordam a gravidez na adolescência. Tem-se como razões para o início tardio da assistência: conhecimento tardio da gravidez, receio em comunicar aos familiares, dificuldade de acesso aos serviços de saúde, constrangimento e medo dos procedimentos durante as consultas, dificuldades para assumir a gestação, conflitos familiares e desconhecimento da importância dessa assistência. ${ }^{15-16}$

Estes dados apontam a necessidade de as equipes da ESF investirem na sensibilização e motivação das gestantes adolescentes para ingresso precoce nos serviços de saúde, e utilização de estratégias para sua captação, como a busca ativa, e, principalmente, estabelecerem 0 acolhimento e criação do vínculo entre gestante-profissional de saúde.

Além de avaliar a utilização do cuidado pré-natal, que se refere à idade gestacional de início da atenção e ao número de consultas de pré-natal, considerou-se importante verificar as ações desenvolvidas no processo do cuidado como a solicitação e realização de exames laboratoriais de rotina, principalmente VDRL e o anti-HIV. Assim, o cumprimento de todos os exames representa um indicador de qualidade da atenção pré-natal. ${ }^{12}$

0 Pacto pela Saúde, aprovado pelo Conselho Nacional de Saúde em 2006, ressalta como uma de suas prioridades a redução das taxas de transmissão vertical do HIV e da sífilis. No Brasil, foram detectados, de 2000 a 2010, mais de 54 mil casos de sífilis congênita e de infecções pelo HIV em gestantes, sendo que, em indivíduos menores de 13 anos de idade, a transmissão vertical é a categoria de exposição predominante, com percentuais acima de $85 \%$ desde 2006 , e a faixa etária de 13 a 24 anos é relevante, visto que em 2009 foram identificados mais de três mil casos de AIDS entre esses adolescentes e jovens. ${ }^{17}$ 
Supõe-se que o início da vida sexual precoce e a inconsistência no uso de preservativos indicam a vulnerabilidade de adolescentes a estas infecções e como estas podem ser prevenidas; é importante a disponibilidade destes exames antes do parto.

A realização de todos os exames de rotina solicitados no pré-natal pela maioria das adolescentes evidencia a ampliação do acesso aos serviços de saúde, e oferece oportunidade de efetivação de exames antes não realizados.

A vacinação antitetânica da gestante deve ser realizada com a vacina dupla tipo adulto (dT) o mais precocemente possível, independente da idade gestacional (IG). ${ }^{10}$ Sua realização constitui indicador que também reflete atenção pré-natal de qualidade e, particularmente, da equipe de enfermagem, pois constitui prática sob responsabilidade dessa categoria profissional. Quanto ao esquema vacinal, a maioria das gestantes foi avaliada, o que indica que a equipe de enfermagem esteve atenta a este cuidado no pré-natal. 0 percentual de adolescentes que recebeu as três doses da vacina dT pode ser atribuído ao fato de serem primigestas e de não buscarem habitualmente a imunização nos serviços de saúde, somente o fazendo quando engravidam ou têm algum problema de saúde.

Além da imunização antitetânica, constatou-se que mais da metade das adolescentes realizaram a citologia oncótica, e todas realizaram ultrassonografia, o que mostra a disponibilidade dos serviços de saúde para realização desses exames. Embora o PHPN não inclua a citologia oncótica como procedimento básico, deve-se levar em consideração que muitas mulheres frequentam os serviços de saúde apenas para o prénatal; portanto, esse momento pode ser ideal, e às vezes único, para programar ou consolidar as orientações e as práticas preventivas para a saúde feminina. ${ }^{5}$

A realização da ultrassonografia durante a gestação constitui uma tecnologia que proporciona determinação da IG, detecção precoce de gestações múltiplas e malformações fetais clinicamente não suspeitas, entre outros. ${ }^{10}$ De acordo com Ministério da Saúde, ${ }^{10}$ a não realização de ultrassonografia durante a gestação não constitui omissão, nem diminui a qualidade do pré-natal. Contudo, qualifica a atenção.

No estudo, registrou-se que os profissionais de saúde estão atentos a esta prática da amamentação, pois a maioria das adolescentes foi submetida ao exame de mamas e recebeu orientação sobre 0 aleitamento materno.

A adolescência constitui etapa evolutiva de grandes modificações corporais, e, acrescidas das referentes à gravidez, a aceitação da prática da amamentação pode ser um processo mais lento. ${ }^{10}$ Tal afirmação é reforçada em estudo sobre a qualidade da atenção à saúde do adolescente no qual foram obtidos índices elevados de desmame precoce. Portanto, 0 preparo para a amamentação deve ser iniciado durante a gestação e, particularmente para a adolescente, ser sistemático e diferenciado. ${ }^{15}$
Com relação à informação sobre o tipo de parto, a maioria das adolescentes não foi orientada sobre as vantagens e desvantagens dos tipos de parto. 0 pré-natal é o momento mais apropriado para a preparação e orientação para o parto. Além disso, no contexto da saúde sexual e reprodutiva, a escolha do tipo de parto constitui um dos principais direitos reprodutivos das mulheres.

Em relação ao profissional que realizou o atendimento pré-natal, observou-se que, em algumas UBS, a consulta médica foi intercalada com a de enfermagem; em outras, a consulta médica foi referenciada pela enfermeira. Para algumas, a gestação foi considerada de risco, e os médicos foram os responsáveis pelo atendimento desse grupo etário. Contudo, verificou-se que a maioria das adolescentes efetuou 0 acompanhamento pré-natal com o mesmo profissional, o que propicia a formação do vínculo entre médico-adolescente e confere qualidade na atenção.

Além da competência e habilidade dos profissionais de saúde, para a atenção pré-natal qualificada, é necessário que a equipe de saúde realize correta e uniformemente os procedimentos clínicos e obstétricos. ${ }^{10}$ Nesse estudo, observouse que os procedimentos desenvolvidos pelos auxiliares ou técnicos de enfermagem, como a medida do peso e aferição da PA, foram executados com maior frequência quando comparados aos realizados pelo médico e/ou enfermeira na consulta de pré-natal, a exemplo da verificação de edema.

\section{CONCLUSÕES}

Diante dos resultados alcançados, conclui-se que é indispensável garantir a qualidade das ações de saúde e de enfermagem durante o processo assistencial, principalmente quando se trata de gestantes e puérperas adolescentes, pois estas vivenciam uma fase de transformações físicas, emocionais, ou mesmo de caráter psicossocial, que envolvem crenças e valores, e ainda vivenciam a chegada de um filho na adolescência.

Considerando a importância desse tema, recomendase captar precocemente as gestantes adolescentes, sensibilizálas sobre a relevância do retorno ao serviço para revisão puerperal, educar permanentemente os profissionais de saúde que assistem estas adolescentes e criar estratégias e campanhas educativas que facilitem o acesso das adolescentes aos serviços de saúde, com objetivo de informá-las, garantir acesso aos métodos contraceptivos, prevenir agravos à saúde e evitar a reincidência da gravidez na adolescência.

Os resultados do estudo apontam a necessidade de melhorar a organização dos serviços de saúde para 0 acolhimento e acompanhamento das adolescentes desde o prénatal até o puerpério por uma equipe multidisciplinar. Implica o estabelecimento de esforço integrado de todos os níveis gestores para a oferta de serviços que garantam acolhimento, informação, aconselhamento, competência profissional, tecnologia apropriada disponível e relacionamento pessoal 
pautado no respeito à dignidade e aos direitos sexuais $\mathrm{e}$ reprodutivos. $^{18}$

Recomenda-se, ainda, a realização de estudos sobre a qualidade da atenção à saúde das adolescentes durante o ciclo gravídico-puerperal, e sugere-se a utilização de métodos quantitativos com maior número amostral, que elaborem uma matriz de avaliação voltada a este grupo etário.

\section{REFERÊNCIAS}

1.World Health Organization- WHO. Pregnant adolescents: delivering on global promises of hope. Genebra; 2006.

2.Ministério da Saúde (BR). Temático Saúde da Família. Brasília(DF): Organização Panamericana da Saúde; 2008.

3.Ministério da Saúde (BR). Portal da Saúde. Notícias. Partos em adolescentes caem em 30\% em dez anos. 2009. [citado 2010 abr 14]. Disponivel em http://portal.saude.gov.br/portal/aplicacoes/noticias/ default.cfm.

4.Koffman, MD, Bonadio IC. Avaliação da atenção pré-natal em uma instituição filantrópica da cidade de São Paulo. Rev Bras Saude Matern Infant. 2005; 5(1): 23-32.

5.Parada, CMGL. Avaliação da assistência pré-natal e puerperal desenvolvidas em região do interior do Estado de São Paulo em 2005. Rev Bras Saude Matern Infant. 2008; 8 (1): 113-24.

6. Tanaka OY, Melo C. Avaliação de Programas de Saúde do Adolescente: um modo de fazer. São Paulo: Edusp; 2001.

7.Donabedian A. La Calidad de la atención médica: definicion y metodos de evaluacion. La Prensa Medica Mexicana, 1984.

8.Instituto Brasileiro de Geografia e Estatística- IBGE . Contagem da população. 2010. [ citado 2011 mai 13].Disponível em: http:// www.censo2010.ibge.gov.br.

9.Departamento de Informática do SUS - DATASUS. Cadastro Nacional de Estabelecimentos. Piauí. 2009. [citado 2010 jul 18]. Disponível em: http://cnes.datasus.gov.br

10.Ministério da Saúde (BR). Secretaria de Atenção à Saúde. Departamento de Ações Programáticas Estratégicas. Área Técnica de Saúde da Mulher. Pré-natal e Puerpério: atenção qualificada e humanizada - manual técnico. Brasília( DF); 2006.

11.Kotelchuck M. Evaluation of the Kessner adequacy of prenatal care index and proposed adequacy of prenatal care utilization index. Am J Public Health. 1994; 84(9): 1414-420.

12.Almeida SDM, Barros MBA. Equidade e atenção à saúde da gestante em Campinas, Brasil. Rev Panam Salud Publica. 2005; 17(1): 15-25.
13.Nagahama EEI, Santiago S M. 0 cuidado pré-natal em hospital universitário: uma avaliação de processo. Cad Saude Publica. 2006; 22(1): 173-79.

14.Goldenberg P, Figueiredo MCT, Silva RS. Gravidez na adolescência, prénatal e resultados perinatais em Montes Claros, Minas Gerais, Brasil. Cad Saude Publica. 2005; 21(4): 1077-086.

15.Metello J, Torgal M, Viana R, Martins L, Maia M, Casal E. Desfecho da gravidez nas jovens adolescentes. Rev Bras Ginecol Obstet. 2008; (30)12: 620-25.

16.Spindola T, Silva LFF. Perfil epidemiológico de adolescentes atendidas no pré-natal de um hospital universitário. Esc Anna Nery. 2009; 13(1): 99-107.

17. Ministério da Saúde (BR). Secretaria de Vigilância em Saúde.Departamento de DST, Aids e Hepatites Virais. Bol Epidemiol. AIDS e DST. 2010; 7(1)

18.Ministério da Saúde (BR). Secretaria de Atenção à Saúde. Saúde integral de adolescentes e jovens: orientações para a organização de serviços de saúde. Brasília(DF); 2007. 